\title{
Use of a laser beam with an oblique angle of incidence to measure the reduced scattering coefficient of a turbid medium
}

\author{
Lihong Wang and Steven L. Jacques
}

\begin{abstract}
A simple and quick approach is used to measure the reduced scattering coefficient $\left(\mu_{\mathrm{s}}{ }^{\prime}\right)$ of a semi-infinite turbid medium having a much smaller absorption coefficient than $\mu_{\mathrm{s}}{ }^{\prime}$. A laser beam with an oblique angle of incidence to the medium causes the center of the diffuse reflectance that is several transport mean-free paths away from the incident point to shift away from the point of incidence by an amount $\Delta \mathrm{x}$. This amount is used to compute $\mu_{s}{ }^{\prime}$ by $\mu_{s}{ }^{\prime}=\sin \left(\alpha_{\mathrm{i}}\right) /(\mathrm{n} \Delta \mathrm{x})$, wheren is the refractive index of the turbid medium divided by that of the incident medium and $\alpha_{i}$ is the angle of incidence measured from the surface normal. For a turbid medium having an absorption coefficient comparable with $\mu_{s}{ }^{\prime}$, a revision to the above formula is made. This method is tested theoretically by Monte Carlo simulations and experimentally by a video reflectometer.
\end{abstract}

Key words: Tissue optics, turbid media, scattering media, scattering coefficient, optical properties.

\section{Introduction}

In both diagnostic and therapeutic applications of light in medicine, it is important to evaluate optical properties of tissues, including the absorption coefficient $\left(\mu_{\mathrm{a}}\right)$ and the reduced scattering coefficient $\left[\mu_{s}{ }^{\prime}=\mu_{s}(1-g)\right]$, where $\mu_{s}$ is the scattering coefficient and $\mathrm{g}$ is the anisotropy factor of scattering. The optical properties can be used to diagnose diseases, to measure tissue metabolic status, or to determine the dosimetry in therapeutic applications of lasers. Measuring optical properties of tissues in vitro can be accomplished by use of several techniques, of which the integrating sphere measurement is the most common. ${ }^{1}$ Measuring optical properties of tissues in vivo is still a challenge.

Wilson et al. ${ }^{2}$ used an optical fiber bundle and $\mathrm{J}$ acques et al. ${ }^{3}$ used a video reflectometer to measure diffuse reflectance of tissues in vivo. Diffusion theory has been used to compute diffuse reflectance to deduce optical properties. Diffusion theory is valid for an infinitely narrow laser beam and for observation points far from the light source, but a laser beam

The authors are with the Laser Biology Research Laboratory, Box 17, The University of Texas M. D. Anderson Cancer Center, 1515 Holcombe Boulevard, Houston, Texas 77030.

Received 5 April 1994; revised manuscript received 8 September 1994.

0003-6935/95/132362-05\$06.00/0.

(1) 1995 Optical Society of America. of a finite size is used for the measurement of diffuse reflectance. Therefore, diffusion theory is only approximately correct. Another method presented by Patterson et al. ${ }^{4}$ uses the falling tail of time-resolved diffuse reflectance to deduce tissue optical properties, but this method requires expensive instrumentation.

Here we introduce a simple method that deduces $\mu_{s}{ }^{\prime}$ very quickly and is independent of the laser beam size if the laser beam has a predefined mirror symmetry and the size of the beam is smaller than the distance between the observation points and the center of the laser beam. For example, a circular flat beam or a circular Gaussian beam has an elliptic spot on the medium surface when the beam is incident at an oblique angle. The mirror symmetry of this elliptic spot about its short axis permits the application of this method to the measurement of $\mu_{\mathrm{s}}$.

\section{Methods and Materials}

\section{A. Hypothesis}

F or a semi-infinite tissue whose $\mu_{\mathrm{a}} \ll \mu_{\mathrm{s}}{ }^{\prime}$, a narrow laser beam with normal incidence can be approximately represented by a buried isotropic point source, as shown in Fig. 1(a). 5-7 The laser beam's diffuse reflectance that is several transport mean-free paths away from the incident point is well approximated by that of such an isotropic point source. The point source is one scattering mean-free path, $1 / \mu_{s}{ }^{\prime}$, bel ow the tissue surface, which is approximately equal to one transport mean-free path, $1 /\left(\mu_{a}+\mu_{s}{ }^{\prime}\right)$, because 

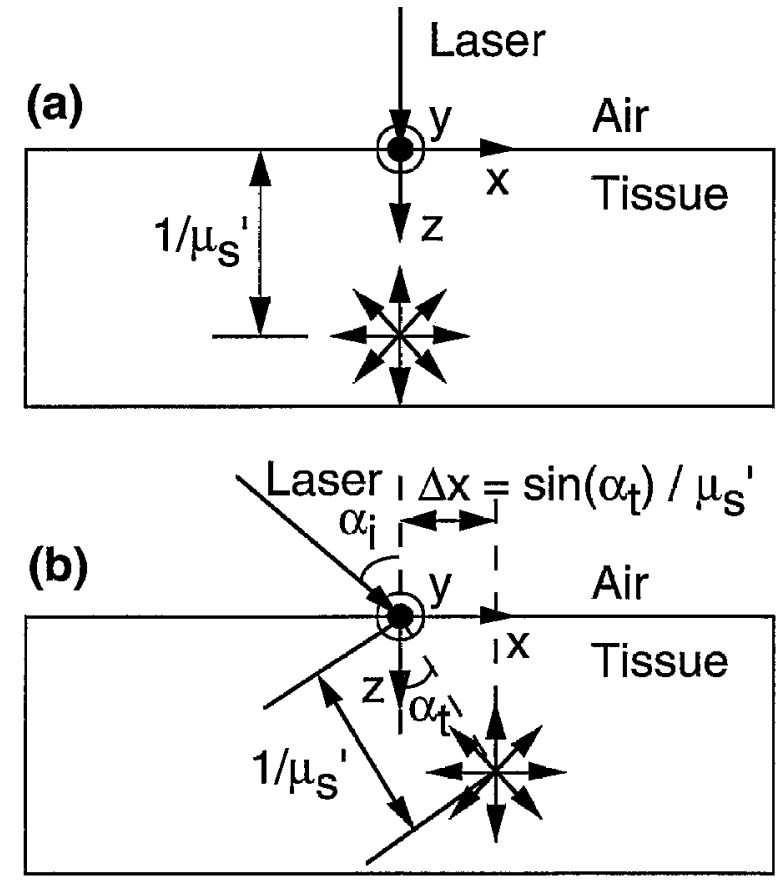

Fig. 1. Lumped isotropic point sources for a laser beam of (a) normal incidence $\left(\alpha_{i}=0\right.$ ), (b) oblique incidence $\left(\alpha_{i}>0^{\circ}\right)$. A coordinate system was set up in which the $y$ axis pointed outward from the paper.

$\mu_{\mathrm{a}} \ll \mu_{\mathrm{s}}{ }^{\prime}$. For a semi-infinite turbid medium with optical properties $\mu_{\mathrm{a}}=0.1 \mathrm{~cm}^{-1}, \mu_{\mathrm{s}}=100 \mathrm{~cm}^{-1}$, and $\mathrm{g}=0.9$, the relative error in diffuse reflectance between the diffusion theory and the Monte Carlo simulation is $<15 \%$ at $x=0.1 \mathrm{~cm}$ and $<10 \%$ for $x>$ $0.2 \mathrm{~cm} .^{6}$ The Monte Carlo simulated results were considered accurate and were used as the standard to evaluate the accuracy of the diffusion theory.

Similarly, a laser beam with oblique incidence can be approximated by an isotropic point source that is $1 / \mu_{\mathrm{s}}^{\prime}$ away from the laser point of incidence al ong the unscattered-light transmission path, which is usually refracted away from the original incident direction [Fig. 1(b)]. As a result, the isotropic point source is horizontally shifted away from the point of incidence by

$$
\Delta \mathrm{x}=\sin \left(\alpha_{\mathrm{t}}\right) / \mu_{\mathrm{s}}{ }^{\prime}=\sin \left(\alpha_{\mathrm{i}}\right) /\left(\mathrm{n} \mu_{\mathrm{s}}{ }^{\prime}\right),
$$

where $\alpha_{i}$ and $\alpha_{t}$ are the angles of incidence and transmission, respectively, and $\mathrm{n}$ is the refractive index of the tissue divided by that of the ambient medium. If the oblique laser beam's diffuse reflectance that is several transport mean-free paths away from the incident point can be approximated by that of the isotropic point source, then the center of this diffuse reflectance will be shifted from the point of incidence by the same amount $\Delta x$. The following subsections describe the testing and revision of Eq. (1) for a better accuracy when $\mu_{\mathrm{a}} \ll \mu_{\mathrm{s}}^{\prime}$ does not hold.

\section{B. Monte Carlo Simulations and Video Reflectometer}

To test Eq. (1) theoretically, we used Monte Carlo modeling ${ }^{8}$ to simulate the diffuse reflectance for a laser beam with various angles of incidence to a semi-infiniteturbid medium of various optical properties. The number of photon packets used for the simulations ranged between 500,000 and 5,000,000, depending on the incident angle and optical properties, such that the computational time for each simulation on a Sun SPARCStation 10 computer did not exceed $24 \mathrm{~h}$. The center line of the diffuse reflectance as a function of $x$ was obtained from the reflectance curve, where the center line consisted of midpoints between the left and right sides of the diffuse reflectance for each reflectance value. The spatial difference between the vertical portion of the center line and the incident point yielded the shift $\Delta \mathrm{x}$. The shift values as a function of the incident angles and optical properties were used to compare with Eq. (1).

To test Eq. (1) experimentally, we used a video reflectometer ${ }^{3}$ to measure the diffuse reflectance from a tissue-simulating turbid medium (Fig. 2). Light from a He-Nelaser (output, $5 \mathrm{~mW}$; wavelength, 632.8 $\mathrm{nm}$ ) was directed to the medium surface at $\alpha_{i}=49^{\circ}$. The 8-bit video CCD camera measured the diffuse reflectance, and the computer collected and then analyzed the CCD image. The dynamic range of the CCD is limited to 255 and is further reduced by the consideration of a good signal-to-noise ratio. Therefore, two images with different intensities of the laser beam weretaken to measure the diffuse reflectance in a wider surface area. Then the diffuse reflectance was analyzed to test Eq. (1).

A proof in Appendix A shows that shift $\Delta x$ is independent of the size of the laser beam if the laser spot on the medium surface has a mirror symmetry about the $y$ axis in Fig. 1 . Of course, the size of the beam should be smaller than the distance between the observation points and the center of the laser beam because the analysis in the hypothesis is valid only for the observation points that are several transport mean-free paths away from the light source. The laser beam for this experiment had an elliptic shape on the surface of the turbid medium and hence

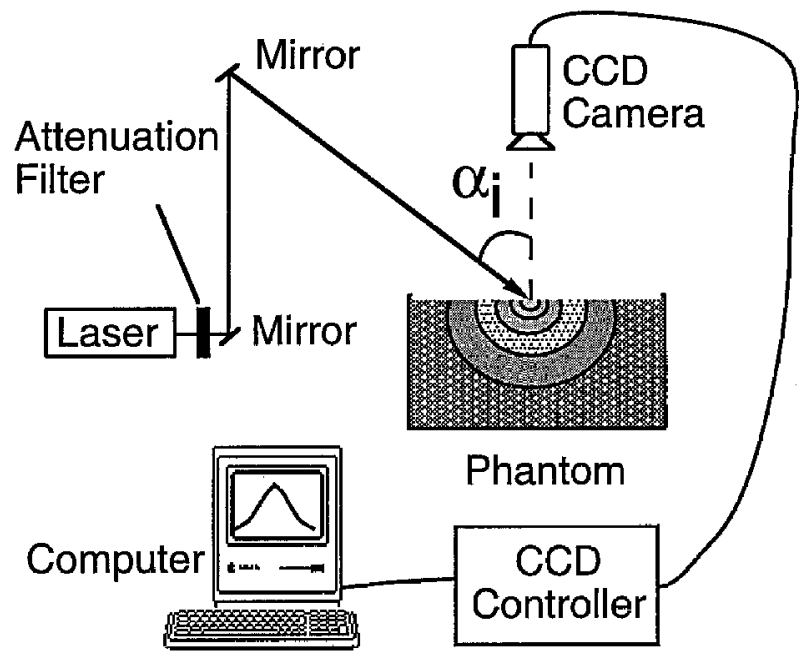

Fig. 2. Schematic of the video reflectometer. 
had a mirror symmetry about the y axis. Therefore this independence validates the above experiment with a laser beam of a finite size instead of an ideal laser beam of an infinitely small size.

\section{Materials}

The tissue-simulating turbid medium consisted of de-ionized water mixed with minimally scattering Trypan blue dye as the absorbers and minimally absorbing polystyrene spheres (diameter, $579 \pm 21$ $\mathrm{nm}$ ) as the scatterers. The $\mu_{\mathrm{a}}$ of the original dye and the $\mu_{\mathrm{s}}$ of the original sphere solution were measured by use of collimated transmission, ${ }^{9}$ and the $g$ of the spheres was computed by use of Mie theory based on the diameter of the spheres and the refractive indices of the spheres (1.56) and of the water (1.33). Then the optical properties of the mixed turbid medium were computed according to the volume concentrations of the two original components in the mixture. The optical properties of the turbid medium for this experiment were $\mathrm{n}=1.33, \mu_{\mathrm{a}}=0.25 \mathrm{~cm}^{-1}, \mu_{\mathrm{s}}=20$ $\mathrm{cm}^{-1}$, and $\mathrm{g}=0.853$. The reduced scattering coefficient was computed to be $\mu_{\mathrm{s}}{ }^{\prime}=2.94 \mathrm{~cm}^{-1}$.

\section{Results}

Figure 3 shows the diffuse reflectance computed with the Monte Carlo model. The optical properties of the turbid medium used in the simulation were chosen to be those of the tissue-simulating turbid medium used in the experiment. The shift based on the vertical portion of curve C was $\Delta \mathrm{x}=0.174 \pm$ $0.009 \mathrm{~cm}$, and the shift based on Eq. (1) and the optical properties was $\Delta \mathrm{x}=0.181 \mathrm{~cm}$. The standard error of the shift computed with the Monte Carlo model was caused by the statistical nature of Monte Carlo simulations. The two values of the shift were in agreement, but the accuracy of $\mathrm{Eq}$. (1) can be improved based on more Monte Carlo simulations, as shown below.

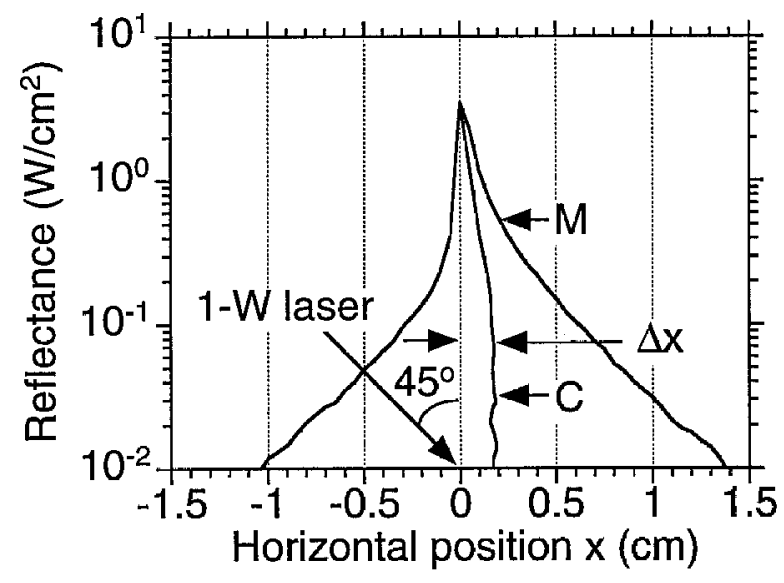

Fig. 3. Curve $M$ is the Monte Carlo simulated diffuse reflectance of a 1-W laser beam incident to a turbid medium with $\alpha_{i}=45^{\circ}$, and curve $C$ is the center line of curve $M$, i.e., the midpoint of the left and right sides of curve $M$ for specific reflectance values. The optical properties of the turbid medium were $n=1.33, \mu_{a}=0.25$ $\mathrm{cm}^{-1}, \mu_{\mathrm{s}}=20 \mathrm{~cm}^{-1}$, and $\mathrm{g}=0.853$.
Figure 4(a) shows the shift values computed by use of the Monte Carlo simulations for various $\mu_{\mathrm{s}}$ values of the turbid media. The dashed curve is based on Eq. (1) and deviates from the Monte Carlo simulated data for small $\mu_{\mathrm{s}}{ }^{\prime}$ values. The deviation was considered to be caused by the increased effect of $\mu_{\mathrm{a}}$ for decreased $\mu_{\mathrm{s}}{ }^{\prime}$ and can be reduced by the addition of the weighted $\mu_{\mathrm{a}}$ to $\mu_{\mathrm{s}}{ }^{\prime}$. From this consideration, the
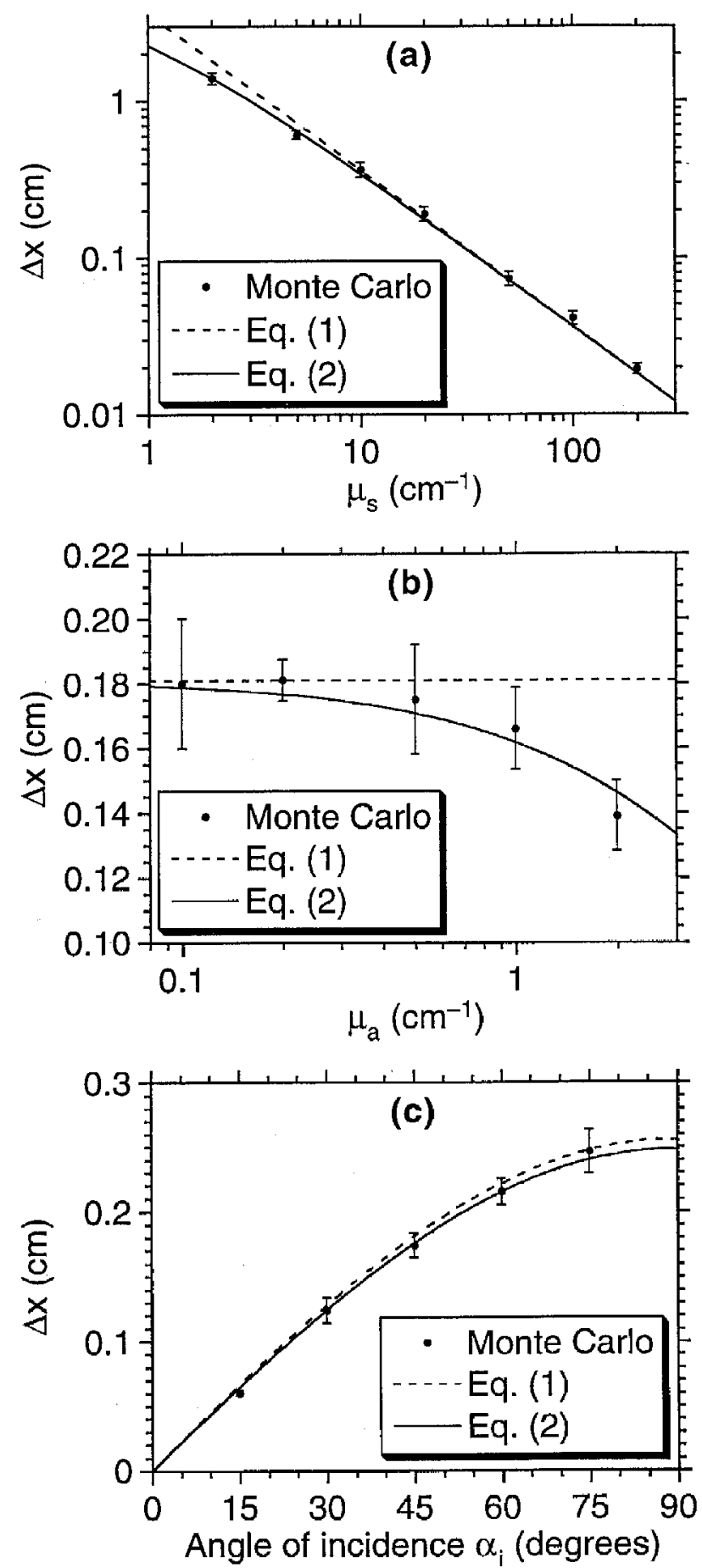

Fig. 4. Symbols with error bars are Monte Carlo simulated $\Delta x$ for different (a) $\mu_{\mathrm{s}}$, (b) $\mu_{\mathrm{a}}$, (c) angles of incidence $\alpha_{\mathrm{i}}$. The dashed and solid curves were computed by use of Eqs. (1) and (2), respectively. The parameters for $(\mathrm{a})-(\mathrm{c})$ are listed in the text. 
computed shift values were found to be fit the best by

$$
\Delta x=\sin \left(\alpha_{\mathrm{i}}\right) /\left[\mathrm{n}\left(\mu_{\mathrm{s}}{ }^{\prime}+0.35 \mu_{\mathrm{a}}\right)\right],
$$

where $\alpha_{\mathrm{i}}=45^{\circ}, \mathrm{n}=1.33, \mu_{\mathrm{a}}=0.25 \mathrm{~cm}^{-1}, \mu_{\mathrm{s}}{ }^{\prime}=$ $\mu_{s}(1-0.853)$, and term $0.35 \mu_{a}$ was introduced to minimize the fitting error. Least-squares fitting was used to determine the coefficient in front of the $\mu_{a}$ in Eq. (2); the fitting correlation coefficient between the raw data and $\mathrm{Eq}$. (2) was 0.999.

Figure 4(b) shows the shift values computed by use of the Monte Carlo simulations for various $\mu_{a}$ values of the turbid media, where $\alpha_{\mathrm{i}}=45^{\circ}, \mathrm{n}=1.33, \mu_{\mathrm{s}}{ }^{\prime}=20$ $\mathrm{cm}^{-1} \times(1-0.853)=2.94 \mathrm{~cm}^{-1}$. Equation (1) fits the data points with low $\mu_{\mathrm{a}}$ values and deviates from those with high $\mu_{a}$ values, whereas Eq. (2) fits the raw data very well.

Figure 4(c) shows the shift values computed by use of the Monte Carlo simulations for various incident angles $\alpha_{i}$. Both Eqs. (1) and (2) fit the raw data well because $\mu_{\mathrm{a}} \ll \mu_{\mathrm{s}}{ }^{\prime}$ in this case, where $\mathrm{n}=1.33, \mu_{\mathrm{a}}=$ $0.25 \mathrm{~cm}^{-1}, \mu_{\mathrm{s}}^{\prime}=20 \mathrm{~cm}^{-1} \times(1-0.853)=2.94 \mathrm{~cm}^{-1}$.

Figure 5 shows the experimental results obtained with the video reflectometer described in Fig. 2. Because the laser beam was oblique to the surface the reflectance pattern was asymmetrical near the point of incidence, but the diffuse reflectance far from the source formed concentric circles [Figs. 5(a) and 5(b)]. Selecting the reflectance values along the $x$ axis of each image, e.g., along the horizontal line crossing from $A$ to $B$ in Fig. 5(a), and replacing the saturated center portion of $\mathrm{Fig}$. $5(\mathrm{a})$ with the corresponding portion in Fig. 5 (b) compensated by the attenuation factor, we plotted the diffuse reflectance as a function of $x$ in Fig. 5(c). The peak reflectance occurred at the center of the incident laser beam and defined the origin of thexaxis in Fig. 5(c). The circular periphery that is several transport mean-free paths away from the incident point defined an apparent center [Figs. $5(a)$ and $5(b)$ that was offset from the origin by $\Delta x$ and yiel ded a vertical center line [Fig. 5(c)] that shared the same physical meaning with the apparent center. The experimental shift $\Delta x$ in Fig. 5(c) was $0.172 \pm$ $0.003 \mathrm{~cm}$. From Eq. (2) the theoretical shift value was computed to be $0.187 \mathrm{~cm}$, where $\alpha_{i}=49^{\circ}, \mathrm{n}=$ 1.33, $\mu_{\mathrm{a}}=0.25 \mathrm{~cm}^{-1}$, and $\mu_{\mathrm{s}}^{\prime}=2.94 \mathrm{~cm}^{-1}$. The relative error between the experimental and theoretical shift values was $8 \%$.

\section{Conclusions and Discussion}

Equation (2) is a refined version of Eq. (1) and reduces to Eq. (1) when $\mu_{\mathrm{a}} \ll \mu_{\mathrm{s}}{ }^{\prime}$. Equation (1) is valid only when $\mu_{\mathrm{a}} \ll \mu_{\mathrm{s}}{ }^{\prime}$, whereas Eq. (2) is valid even when $\mu_{\mathrm{a}}$ is comparable with $\mu_{\mathrm{s}}{ }^{\prime}$. The computed shift value in Fig. 3 was $0.176 \mathrm{~cm}$ when based on Eq. (2) instead of $0.181 \mathrm{~cm}$ when based on Eq. (1), and the value of $0.176 \mathrm{~cm}$ was in better agreement with the shift value $0.174 \pm 0.009 \mathrm{~cm}$ computed with the Monte Carlo simulations.

Equation (2) fits the Monte Carlo simulation results in Fig. 4 very well for different optical properties and incident angles and agreed with the video reflectometry measurement with an $8 \%$ error. The experi-
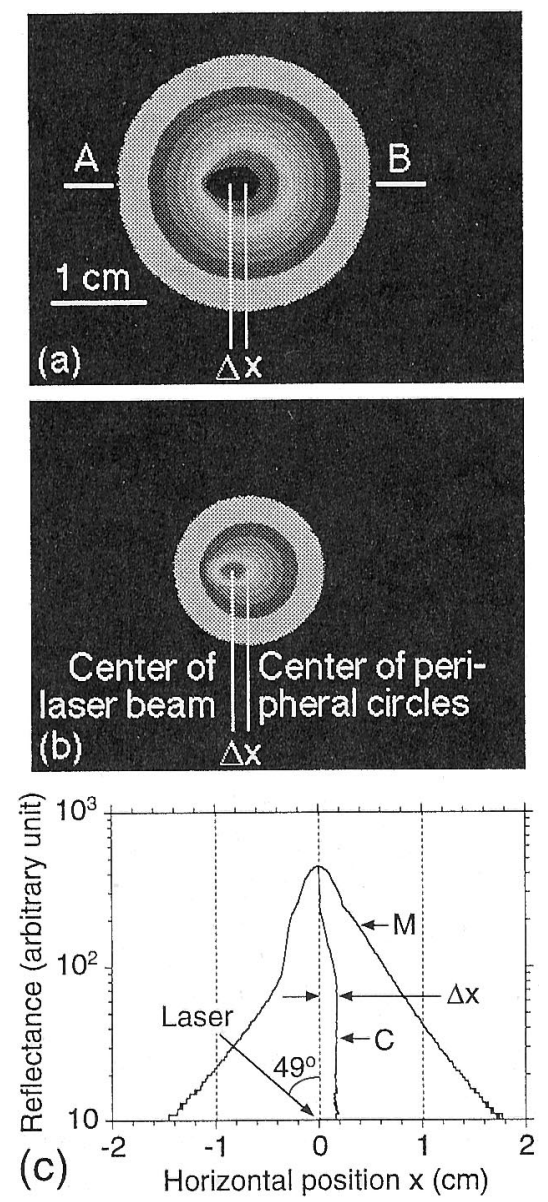

Fig. 5. (a), (b) CCD video images of a diffuse reflectance pattern from a turbid medium with the same optical properties as those in Fig. 3; (c) diffuse reflectance along the xaxis. The resolution of the images was $8.5 \times 10^{-3} \mathrm{~cm} / \mathrm{pixel}$; (a) is the video image without laser beam attenuation (the center of the image was saturated on the CCD camera) and (b) is the video image with a 3.3-fold attenuation by a filter to measure the saturated center portion of (a).

ment used an 8-bit CCD camera, and two measurements with and without the attenuation of the laser source intensity were required. The neutral-density filter may shift the center of the laser beam slightly. The difference between the theoretical and experimental shift values was $0.015 \mathrm{~cm}$, which was only 1.8 pixels on the CCD camera because the resolution in the setup was $8.5 \times 10^{-3} \mathrm{~cm} / \mathrm{pixel}$. Therefore the accuracy of the experiment was limited by the resolution and the dynamic range of the camera.

When $\mu_{\mathrm{a}} \ll \mu_{\mathrm{s}}{ }^{\prime}$, e.g., $\mu_{\mathrm{a}}=0.1 \mathrm{~cm}^{-1}$ and $\mu_{\mathrm{s}}{ }^{\prime}=10 \mathrm{~cm}^{-1}$ which are typical for biological tissues at visible wavel engths ${ }^{10}$ ), Eq. (2) is reduced to the simpler Eq. (1). Because $\alpha_{i}$ and $n$ are known, Eq. (1) suggests that if we measure the diffuse reflectance of a laser beam with oblique incidence and compute $\Delta x$, then we can calculate $\mu_{s}^{\prime}$ by using

$$
\mu_{\mathrm{s}}{ }^{\prime}=\sin \left(\alpha_{\mathrm{i}}\right) /(\mathrm{n} \Delta \mathrm{x}) .
$$

Equation (9) provides a simple approach for measuring the reduced scattering coefficient that can be 
applied to biological tissues for diagnosis of disease in clinical settings.

When $\mu_{\mathrm{a}}$ is comparable with $\mu_{\mathrm{s}}{ }^{\prime}$, then $\mu_{\mathrm{s}}{ }^{\prime}$ cannot be solved from the measurement of $\Delta x$ independently. Another technique may be used in combination with this method to determine both $\mu_{\mathrm{a}}$ and $\mu_{\mathrm{s}}{ }^{\prime}$. For example, the falling tail of time-resolved diffuse reflectance can be used to determine $\mu_{\mathrm{a}}{ }^{4}$ In summary, the validity of this approach that is used to measure reduced scattering coefficients of turbid media was theoretically and experimentally tested. The approach is straightforward and quick, thus having potential for clinical applications.

\section{Appendix A}

Here we present a mathematical proof that shows that the shift value is independent of the size of the incident laser beam if the beam has a mirror symmetry about the $y$ axis (Fig. I) and if the size of the laser beam is smaller than the distance between the observation points and the incident point. Assuming that an infinitely narrow laser beam's diffuse reflectance that is several transport mean-free paths away from the incident point, $G(x, y)$, has a mirror symmetry about $x=\Delta x$ (Fig. 1), i.e., $G(\Delta x-x, y)=G(\Delta x+x, y)$, we find that if a laser beam of a finite size with an intensity profile $S(x, y)$ has a mirror symmetry about the $y$ axis, i.e., $S(-x, y)=S(x, y)$, then this finite-size laser beam's diffuse reflectance that is several transport mean-free paths away from the incident point, $R(x, y)$, will also have a mirror symmetry about $x=\Delta x$.

Because the turbid medium is semi-infinite, $R(x, y)$ can be expressed with a convolution, ${ }^{11}$

$$
R(x, y)=\int_{-\infty}^{+\infty} \int_{-\infty}^{+\infty} S\left(x^{\prime}, y^{\prime}\right) G\left(x-x^{\prime}, y-y^{\prime}\right) d x^{\prime} d y^{\prime}
$$

and we obtain

$$
\begin{aligned}
R(\Delta x-x, y)= & \int_{-\infty}^{+\infty} \int_{-\infty}^{+\infty} S\left(x^{\prime}, y^{\prime}\right) \\
& \times G\left(\Delta x-x-x^{\prime}, y-y\right) d x^{\prime} d y^{\prime}, \\
= & \int_{-\infty}^{+\infty} \int_{-\infty}^{+\infty} S\left(x^{\prime}, y^{\prime}\right) \\
& \times G\left(\Delta x+x+x^{\prime}, y-y\right) d x^{\prime} d y^{\prime} \\
= & \int_{+\infty}^{-\infty} \int_{-\infty}^{+\infty} S\left(-x^{\prime}, y^{\prime}\right) \\
& \times G\left(\Delta x+x-x^{\prime}, y-y\right) d\left(-x^{\prime}\right) d y^{\prime}, \\
= & \int_{-\infty}^{+\infty} \int_{-\infty}^{+\infty} S\left(x^{\prime}, y^{\prime}\right) \\
& \times G\left(\Delta x+x-x^{\prime}, y-y\right) d x^{\prime} d y^{\prime}, \\
= & R(\Delta x+x, y),
\end{aligned}
$$

which means that $\mathrm{R}(\mathrm{x}, \mathrm{y})$ has a mirror symmetry about $\mathrm{x}=\Delta \mathrm{x}$.

We thank L. Eppich for proofreading the manuscript. This research was supported in part by the Whitaker Foundation, U.S. Office of Naval Research grant N00014-91-J -1354, U.S. Air F orce Office of Scientific Research grant F 49620-93-1-0298DEF, U.S. Department of Energy grant DE-FG05-91ER61226, and National Institutes of Health grant R29HL45045.

\section{References}

1. J. W. Pickering, S. A. Prahl, N. Vanwieringen, J. F. Beek, H. J . C. M. Sterenborg, and M. J . C. van Gemert, "Doubleintegrating-sphere system for measuring the optical properties of tissue,"Appl. Opt. 32, 399-410 (1993).

2. B. C. Wilson, T. J . Farrell, and M. S. Patterson, "An optical fiber-based diffuse reflectance spectrometer for noninvasive investigation of photodynamic sensitizers in vivo," in Future Directions and Applications in Photodynamic Therapy, C. J . Goner, ed., Vol. IS06 of SPIE Institute Series (Society of Photo-Optical Instrumentation Engineers, Bellingham, Wash., 1990), pp. 219-232.

3. S. L. J acques, A. Gutsche, J . A. Schwartz, L.-H. Wang, and F. K. Tittel, "Video reflectometry to extract optical properties of tissue in vivo," in Medical Optical Tomography: Functional Imaging and Monitoring, ISII of SPIE Institute Series (Society of Photo-Optical Instrumentation Engineers, Bellingham, Wash., 1993), pp. 211-226.

4. M. S. Patterson, B. Chance, and B. C. Wilson, "Time-resolved reflectance and transmittance for the noninvasive measurement of tissue optical properties," Appl. Opt. 28, 2331-2336 (1989).

5. T. J . Farrell, M. S. Patterson, and B. C. Wilson, "A diffusion theory model of spatially resolved, steady-state diffuse reflectance for the noninvasive determination of tissue optical properties in vivo," Med. Phys. 19, 879-888(1992).

6. L.-H. Wang and S. L. J acques, "Analysis of diffusion theory and similarity relations," in Photon Migration and I maging in Random Media and Tissues, R. R. Alfano and B. Chance, eds., Proc. Soc. Photo-Opt. Instrum. Eng. 1888, 107-116 (1993).

7. L.-H. Wang and S. L. J acques, "Hybrid model of Monte Carlo simulation and diffusion theory for light reflectance by turbid media,"J . Opt. Soc. Am. A 10, 1746-1752 (1993).

8. L.-H. Wang and S. L. J acques, "Animated simulation of light transport in tissues," in Laser-Tissue Interaction 5, S. L. J acques, ed., Proc. Soc. Photo-Opt. Instrum. Eng. 2134A, 247-254 (1994).

9. S. T. Flock, S. L. J acques, B. C. Wilson, W. M. Star, and M. J . van Gemert, "Optical properties of intralipid: a phantom medium for light propagation studies," Lasers Surg. Med. 12, 510-519 (1992).

10. W. F. Cheong, S. A. Prahl, and A. J. Welch, "A review of the optical properties of biological tissues," IEEE J. Quantum Electron. 26, 2166-2185 (1990).

11. S. A. Prahl, M. Keijzer, S. L. J acques, and A. J. Welch, "A Monte Carlo model of light propagation in tissue," in Dosimetry of Laser Radiation in Medicineand Biology, G. J . Mueller and D. H. Sliney, eds., Vol. IS05 of SPIE Institute Series (Society of Photo-Optical Instrumentation Engineers, Bellingham, Wash., 1989), pp. 102-111. 